\title{
2-D inversion of VES data in Saqqara archaeological area, Egypt
}

\author{
Gad El-Qady, Chika Sakamoto, and Keisuke Ushijima \\ Engineering Geophysics, Faculty of Engineering, Kyushu University, 36 Hakozaki, Fukuoka 812-8581, Japan
}

(Received October 14, 1998; Revised August 16, 1999; Accepted August 23, 1999)

\begin{abstract}
The interpretation of actual geophysical field data still has a problem for obtaining a unique solution. In order to investigate the groundwater potentials in Saqqara archaeological area, vertical electrical soundings with Schlumberger array have been carried out. In the interpretation of VES data, 1D resistivity inversion has been performed based on a horizontally layered earth model by El-Qady (1995). However, some results of 1D inversion are not fully satisfied for actual 3D structures such as archaeological tombs. Therefore, we have carried out 2D inversion based on ABIC least squares method for Schlumberger VES data obtained in Saqqara area. Although the results of 2D cross sections were correlated with the previous interpretation, the 2D inversion still shows a rough spatial resistivity distribution, which is the abrupt change in resistivity between two neighboring blocks of the computed region. It is concluded that 3D interpretation is recommended for visualizing ground water distribution with depth in the Saqqara area.
\end{abstract}

\section{Introduction}

Inversion of geophysical data involves the estimation of the parameters of a postulated earth model from a set of observed data. It may be viewed as an attempt to fit the response of an idealized subsurface earth model to a finite set of observed data. With the increased availability of faster computers, it is now practical to employ numerical modeling techniques to invert resistivity data for a 3D geological structure. However, there has been no great success in overcoming the uniqueness problem associated with the practical; that is uncertain, incomplete, geophysical data. The Monte Carlo method, in which a huge number of randomly generated models are tested against the data, has been used for resistivity soundings data (Strenberg, 1979) in an attempt to characterize all models which agree with the observations. Such computations can never be exhaustive, and even calculation ranging over the class of simple layered models is computationally extravagant in the light of the insight obtained from them. The best policy may be to seek a model whose features are in some way essential characteristics of any of the possible solutions, one of which presumably is the true structure, (Vozoff and Jupp, 1975; Constable et al., 1987).

However, the dimensionality of the earth's model should be based on the general characteristics of the geology within the survey region. This dimensionality is often to be determining simply by the availability of the interpretation techniques. Whatever, the basic motivation for seeking smooth model is that we do not wish to be misled by features that appear in the model but are not essential in matching the observations.

In this study, we carried out 2D inversion using the algorithm proposed by Uchida (1991). Since the forward prob-

Copy right (C) The Society of Geomagnetism and Earth, Planetary and Space Sciences (SGEPSS); The Seismological Society of Japan; The Volcanological Society of Japan; The Geodetic Society of Japan; The Japanese Society for Planetary Sciences. lem of electrical and electromagnetic fields for a given earth model is usually non-linear problems, we have to linearize the problem by a certain assumption to perform the least squares inversion. A Jacobian matrix, consisting of partial derivatives of the data with respect to the model parameters, is often ill conditioned; hence the direct solution of the matrix provides a non-realistic rough resistivity model of a large oscillation.

\section{Theoretical Basis}

Here in this algorithm, we consider a two-dimensional earth model whose resistivity varies along the $x$ - and $z$-axis and doesn't change along $y$-axis. Since the current is injected at a point source on the ground surface, however it flows three dimensionally in the earth. The response in a 2D earth is given by Poisson's equation as:

$$
-\nabla \cdot[\sigma(x, z) \nabla V(x, y, z)]=I(x, y, z),
$$

where $\sigma(x, z)$ is the conductivity, $V(x, y, z)$ is the electric potential and $I(x, y, z)$ represents the current source intensity. By applying the Fourier transform to Eq. (1) with respect to the $y$ coordinate, we obtain:

$$
\begin{aligned}
-\nabla & {\left[\sigma(x, z) \nabla \hat{V}\left(x, k_{y}, z\right)\right]+k_{y} \sigma(x, z) \hat{V}\left(x, k_{y}, z\right) } \\
& =\hat{I}\left(x, k_{y}, z\right),
\end{aligned}
$$

where ${ }^{\wedge}$ means the Fourier transform and $k_{y}$ is the Fourier transform variable. A detailed explanation of the finite element discretization of Eq. (2) is given in Sasaki (1981). Discretization over mesh yields a matrix equation,

$$
K V=S
$$

where $K$ is a $L \times L$ sparse band matrix with positive symmetric values. This is determined by the geometry and conductivity of each finite element, $L$ is the number of nodes, $V$ is a column vector of the unknown potential at each node, 
and $S$ is the column vector of current source intensity at each node. The potential $V$ in real 3D domain can be obtained by solving Eq. (3) and applying inverse Fourier transform,

$$
\Delta V(x, 0, z)=(1 / \pi) \int_{0}^{\infty} \hat{V}\left(x, k_{y}, z\right) d k_{y},
$$

and the apparent resistivity for Schlumberger can be calculated as:

$$
\rho_{a}=\frac{G \Delta V}{I},
$$

where $G$ is the geometrical factor, and $\Delta V$ is the calculated potential difference between the receiving electrodes, $\boldsymbol{M}$ and $N$.

If we let $\left(P_{i}\right)$ be 2D model's parameters, $\left(\rho_{a j}\right)$ be apparent resistivities, $(\phi)$ the misfit between the observed and the theoretical apparent resistivities. The improvement of parameters of the model should be expressed in a logarithmic scale as:

$$
\begin{aligned}
x_{i} & =\log P_{i}, & i & =1, \ldots, m \\
y_{j} & =\log \rho_{a j}, & j & =1, \ldots, n
\end{aligned}
$$

where: $m$, is the number of model parameters, and $n$, is the number of the observed data.

Here we describe the 2D model, which holds its block boundaries during the inversion and only the resistivity within each block changes with the iteration procedure. The misfit $(\phi)$ of the data is determined by the equation:

$$
\phi=\frac{1}{n} \sum_{j=1}^{n}\left\{y_{j}^{o}-y_{j}^{c}(x)\right\}^{2},
$$

where $\left(y_{j}^{o}\right)$ is the observed data and $\left(y_{j}^{c}\right)$ is the calculated data. If we consider the $(k-1)$-th iteration of the inversion process, applying the Taylor expansion to $y_{j}(x)$ at $x^{(k-1)}$ and neglect the terms of the second and higher order we obtain:

$$
y_{j}\left(x^{(k)}\right)=y_{j}\left(x^{(k-1)}\right)+\sum_{i=1}^{m}\left(\frac{\partial y_{j}}{\partial x_{i}}\right)_{x^{(k-1)}} \Delta x_{i}^{k},
$$

where the superscript $(k)$ means $k$-th iteration. In order to minimize the misfit $\phi$, the condition $\frac{\partial \phi}{\partial x_{i}}=0$ should be satisfied. Furthur explanation was descried by Uchida (1991).

In order to judge the convergence, a statistical criterion, ABIC (Akaike Bayesian Information Criterion) has been proposed by applying the maximum entropy theorem to the Bayesian statistical (Akaike, 1980). This ABIC works as an index to determine the maximum likelihood of the model. A smaller ABIC indicates a larger likelihood and entropy, hence gives a best model. The model is obtained under the assumption that the data error and roughness (spatial derivatives of the parameters) are normally distributed with zeromean. The optimum smoothness is also obtained in the process of the likelihood maximization.

For the least-squares inversion with smoothness regularization, we seek a model, which minimizes both the data misfit and model roughness. From a statistical point of view, the Bayesian procedure can be applied for this purpose by taking the smoothness constraint as a prior distribution of the model parameters (Jackson and Matsura, 1985). One of the advantages of this method is that we do not always require information on individual measurement error to judge the convergence. Also, the selection of an optimum smoothness is completely objective. According to Akaike (1980), bayesian likelihood can be expressed as follows:

$$
L(m / d)=\int p(d / m) \pi(m) d m,
$$

where, $p(d / m)$ is the probability density function of the data, which is called the data distribution, and $\pi(m)$ is a prior distribution. In Bayes'rule, we assume that the model is based on a priori information, such as a smoothness constraint or a parameter constraint, and that its probability density function is $\pi(m)$. It is supposed that the model which maximizes the bayesian likelihood in Eq. (9) makes the average logarithmic likelihood maximum. ABIC is derived to provide an index for finding the maximum Bayesian likelihood and defined by:

$$
\begin{aligned}
A B I C= & (-2) \log (\max L(m / d)) \\
& +2 \operatorname{dim}(\text { hyperparameter })
\end{aligned}
$$

where $d$ is a set of observed data and a hyperparameter means a parameter, which is not used to express the model directly, but is used to obtain parameters of the model. The only hyperparameter in this case is the smoothing parameter $(\alpha)$, further explanation of the equations was described by Uchida (1993), then ABIC can be written as:

$$
\begin{aligned}
A B I C(\alpha)= & n \log \left(2 \pi \frac{U}{n}\right)-\log \left|\alpha^{2} C^{T} C\right| \\
& +\log \left|(W A)^{T}(W A)+\alpha^{2} C^{T} C\right| \\
& +n+2
\end{aligned}
$$

where: $A$ is Jacobian matrix, defined by $A_{i j}=\partial y_{i} / \partial \rho_{j}, m$, is a hypothetical model, $W$, is a diagonal weighting, $U$ is a function defined as:

$$
\begin{aligned}
U & =\text { misfit }+ \text { roughness penality of the model } \\
& =\|W d-W F(m)\|^{2}+\alpha^{2}\|C m\|^{2},
\end{aligned}
$$

$C$, is a roughness matrix of a model parameter, which gives the finite difference of the model parameters between laterally and vertically adjacent blocks in the mesh, and $F$ is a non-linear forward function which works on the model to obtain the response.

As well as the optimum smoothness is judged by minimizing $\mathrm{ABIC}$, we have to consider weighted root mean square misfit as well as another misfit parameter called u-rms. This u-rms misfit is defined as:

$$
u \text {-rms misfit }=\sqrt{\frac{U}{n-1}} .
$$

\section{Geologic Setting}

The geologic setting of Saqqara area had been studied by different authors such as Said (1975 and 1990). Topographically, it is a relatively low relief with altitudes varying from 30 to $80 \mathrm{~m}$ above sea level. Geomorphologically, it comprises of flood plain, flood plain fringes, the pediment plain 


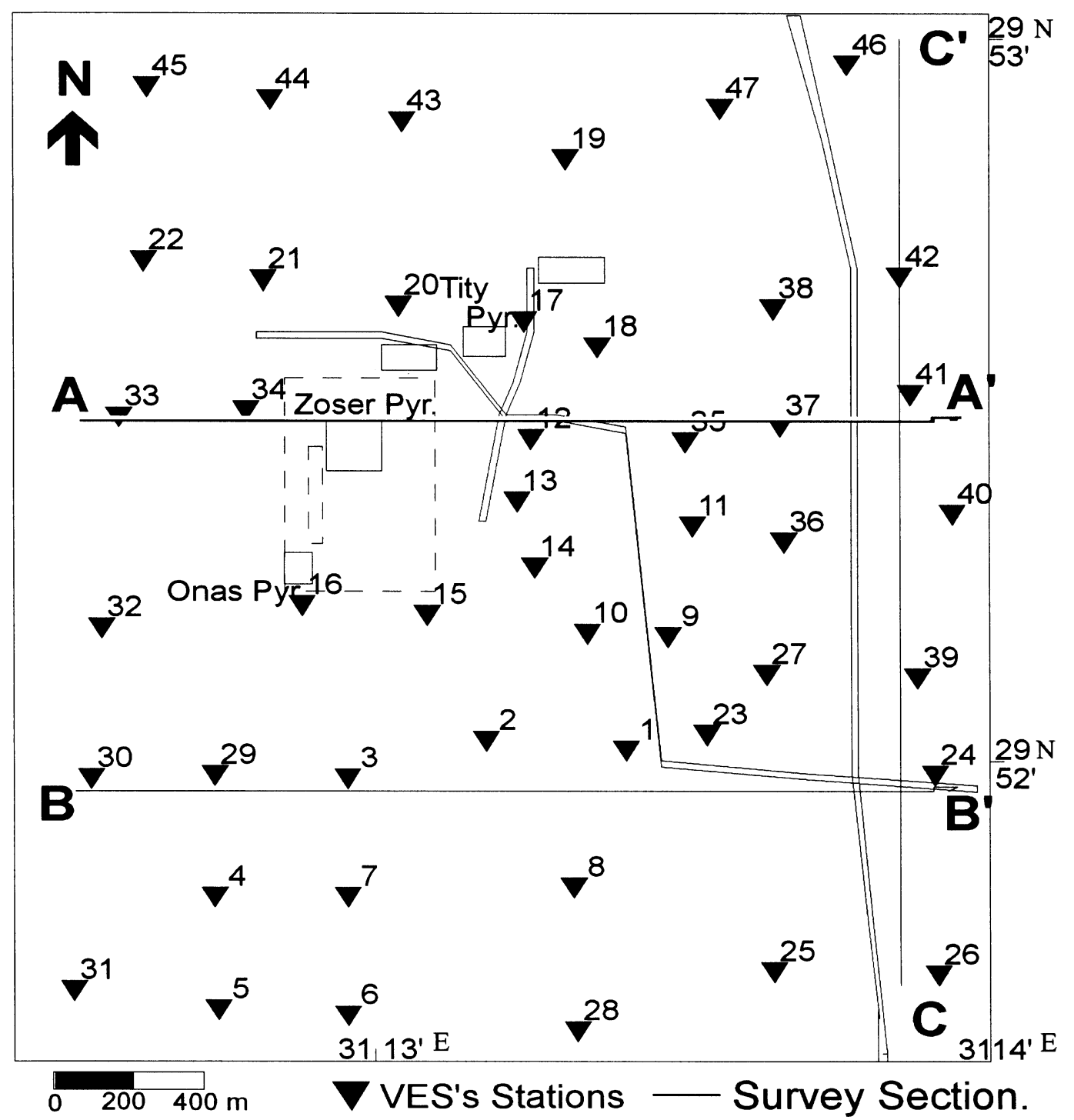

Fig. 1. Location Map for Geophysical survey in Saqqara area.

which extends between Saqqara plateaus and the Nile flood plain and the plateau, where Saqqara pyramids are found.

Lithostratigraphically, the shallow geologic section of Saqqara area composed of Quaternary deposits which comprises of river terraces of sands and gravel with a thickness varying between 30 to $180 \mathrm{~m}$. These were underlined by gravel units $(10 \mathrm{~m})$ of Pleistocene, which underlined by Pliocene formations. Pliocene rocks are varying in composition from limestone to marl and sandstone with total thickness about $23 \mathrm{~m}$. This unit overlaps the Eocene formations with angular unconformity. Eocene rocks are represented by a sandy to marly yellow limestone. These rocks uncomformably lie over the Cretaceous limestone rocks.

\section{Data Analysis and Interpretation}

In this work, forty-seven vertical electrical soundings (VES) were conducted in a grid pattern aiming to map the groundwater aquifer in Saqqara area (Fig. 1). The wellknown Schlumberger configuration of $\mathrm{AB} / 2$ starting with 1 meter up to 300 meter in successive steps was applied.
At few stations measurements were found convenient to be ended before $300 \mathrm{~m}$. The distance between stations varies between 300 and 500 meter according to the topography, land feasibility and the applicability of the array. A 1D interpretation had been done by (El-Qady, 1995) using Zohdy's (1989) method. The main resistivity regime is of the type AAHA $\left(\rho_{1}>\rho_{2}>\rho_{3}>\rho_{4}<\rho_{5}<\rho_{6}\right)$ and their lithologies vary between gravel, marly sand, sandy clay, saturated sand and limestone respectively. The form of the results automatically reflects the locations and the electrical properties of relevant layers buried in the earth. The method is robust and requires no external information on the number of high and low of the resistivity function. So, it is important to understand how and to what extent 1D approximations represent the true structure. This will be done using multidimensional inversion methods such as $2 \mathrm{D}$ or $3 \mathrm{D}$.

In this work, we present 2D inversion for the same data set using Uchida's (1991) algorithm. The algorithm had been modified by the staff of Exploration Geophysics Lab., Kyushu University. We made some changes in calculating 


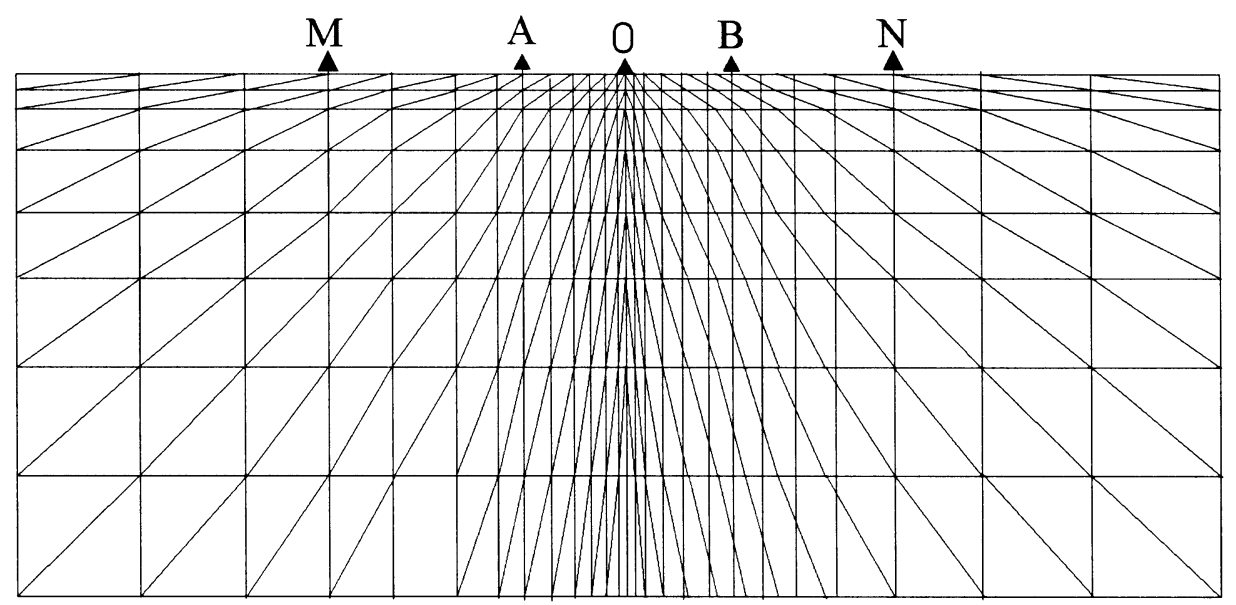

Fig. 2. Shematic view of the calculation mesh.

mesh parameters to be compatible with our $\mathrm{AB} / 2$ distances (Fig. 2). That is one of the advantages of this algorithm that we can handle almost three decades of $A B / 2$ with a single calculation mesh. In the forward calculation, the current electrodes were put near the center point and potential electrodes at outer position. In this case, due to the reciprocity theorem, apparent resistivity for all $\mathrm{AB} / 2$ of all the sounding can be obtained by one forward calculation.

\subsection{Inversion results}

As we seek a model which minimizes both the data misfit and model roughness, and whenever the selection of an optimum smoothness is completely objective. So, we have to run the inversion process until the best fit is attained. The program outputs 7 models per iteration, accompanied by 7 values for all the inversion parameters. Then it selects the best model according to the smoothing factor and rms-misfit as an initial model for the next iteration. Figure 3 represents rms- misfit and the smoothing parameter $(\alpha)$ as a function of the iteration number for the profiles A-A', B-B' and C-C' respectively. It is obvious that both of rms. and $\alpha$ nearly converge with a similar manner; decrease as iteration proceeds. We can judge that, after the fifth iteration, they become nearly stable with minimum values.

Since we have 7 models per iteration, we should investigate the behavior of the inversion parameters (ABIC, u-rms, roughness, and $\alpha$ ) for each iteration as shown in Fig. 4 for the profile (A-A'). At the first iteration, all the inversion parameters have higher values that indicate a non-smooth model. Whereas the iteration process proceeds, the parameters curve become smaller amplitude and slightly smoother. The decision of the convergence can be done by checking how ABIC decreases as the iteration proceeds. When the convergence is attained, the decreases per iteration become very small as shown in Fig. 4(a). It is obvious that rms. and Urms misfits also converge with a similar manner and attain the minima at the fifth iteration. This is the same with the roughness parameter. Figure 5 shows the inversion parameters as a function of the smoothing parameter $(\alpha)$ at each iteration for the profile B-B'. In the first iteration all the parameters have higher values which decrease as the iteration proceeds. Also, the minima of $\mathrm{ABIC}$, rms and Urms misfits are obvious for
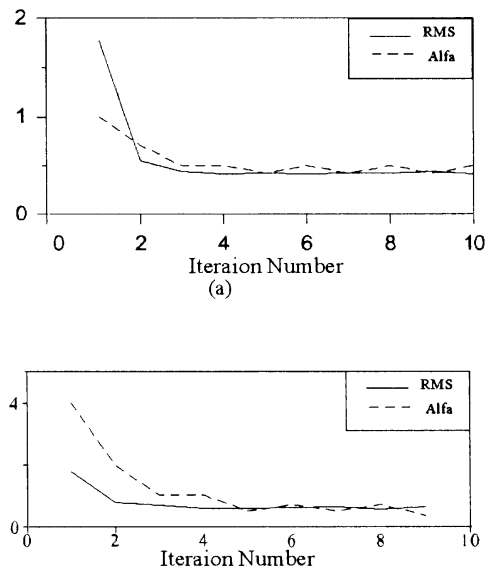

(b)

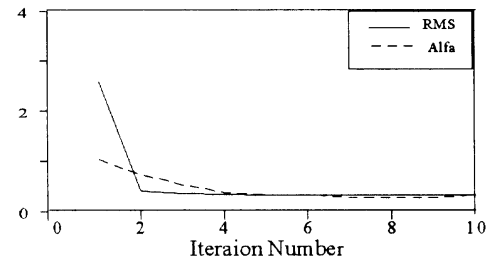

(c)

Fig. 3. The rms misfit and Alfa as a function of iteration numbers: (a) the profile (A-A'), (b) the profile (B-B') and (c) the profile (C-C').

the first three iterations. However, for the all iterations, a rougher model gives a smaller misfit, while the minima of the curve is still clear except for the fifth iteration, it becomes nearly straight line as shown in Figs. 5(a), (b) and (d).

\section{2 $2 \mathrm{D}$ cross sections}

Depending on the results and variation of the inversion parameters, we can conclude that the model of the fifth iteration represents the best fit and minimum $\mathrm{ABIC}$ for this data set.

Figure 6 shows 2D cross section of the inverted model results after the fifth iteration of the profile A-A'. However we have tested the initial homogeneous earth model as 10, 30, 100 and $500 \Omega \mathrm{m}$, it was found that the $100 \Omega \mathrm{m}$ is the best initial model for this data set from the fitting point of view. The topography is incorporated in the modeling. The smoothing factor is 0.4 , while the number of observed data points used 

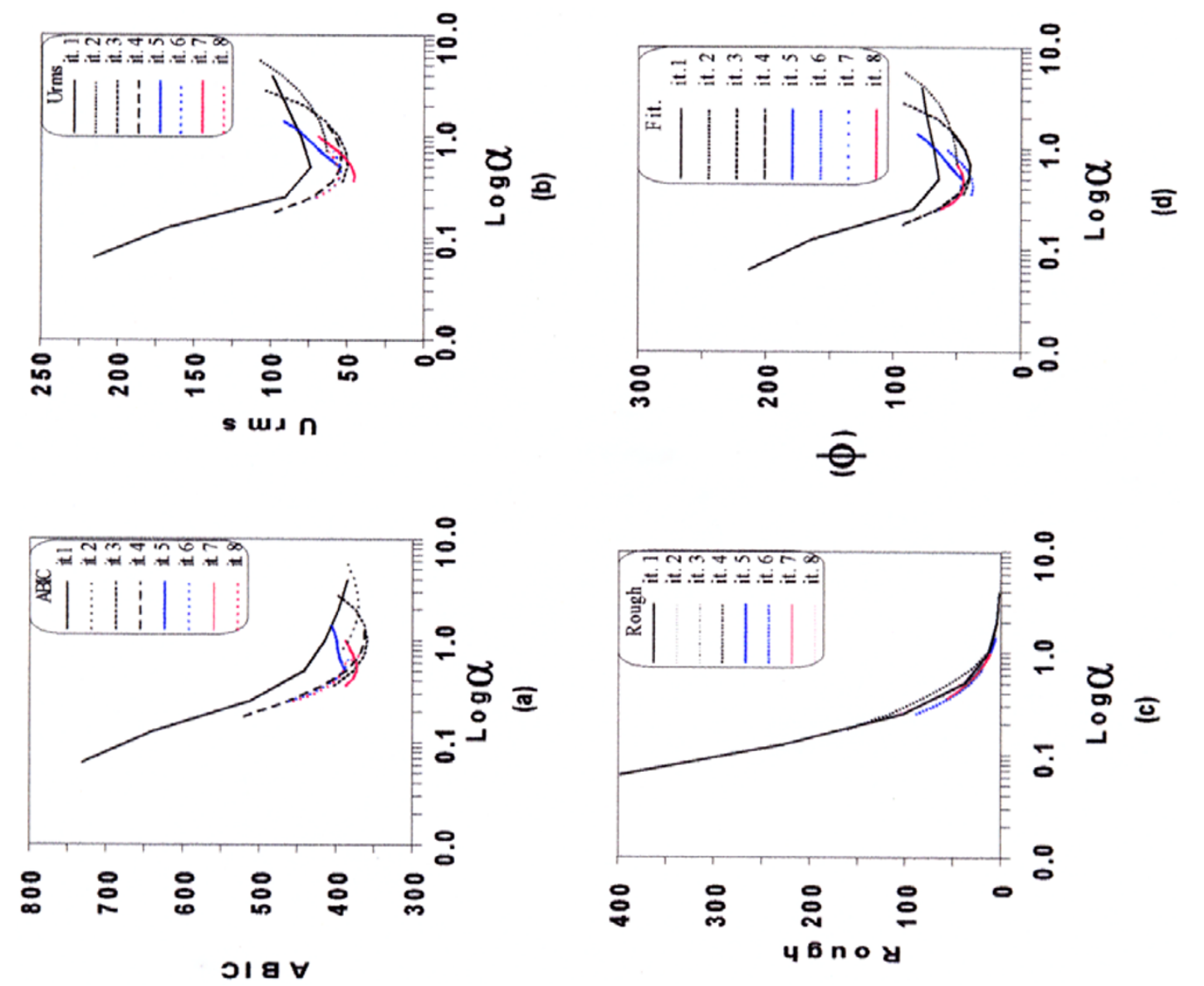

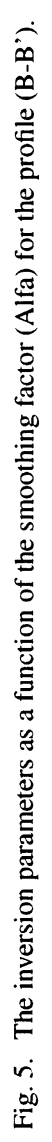
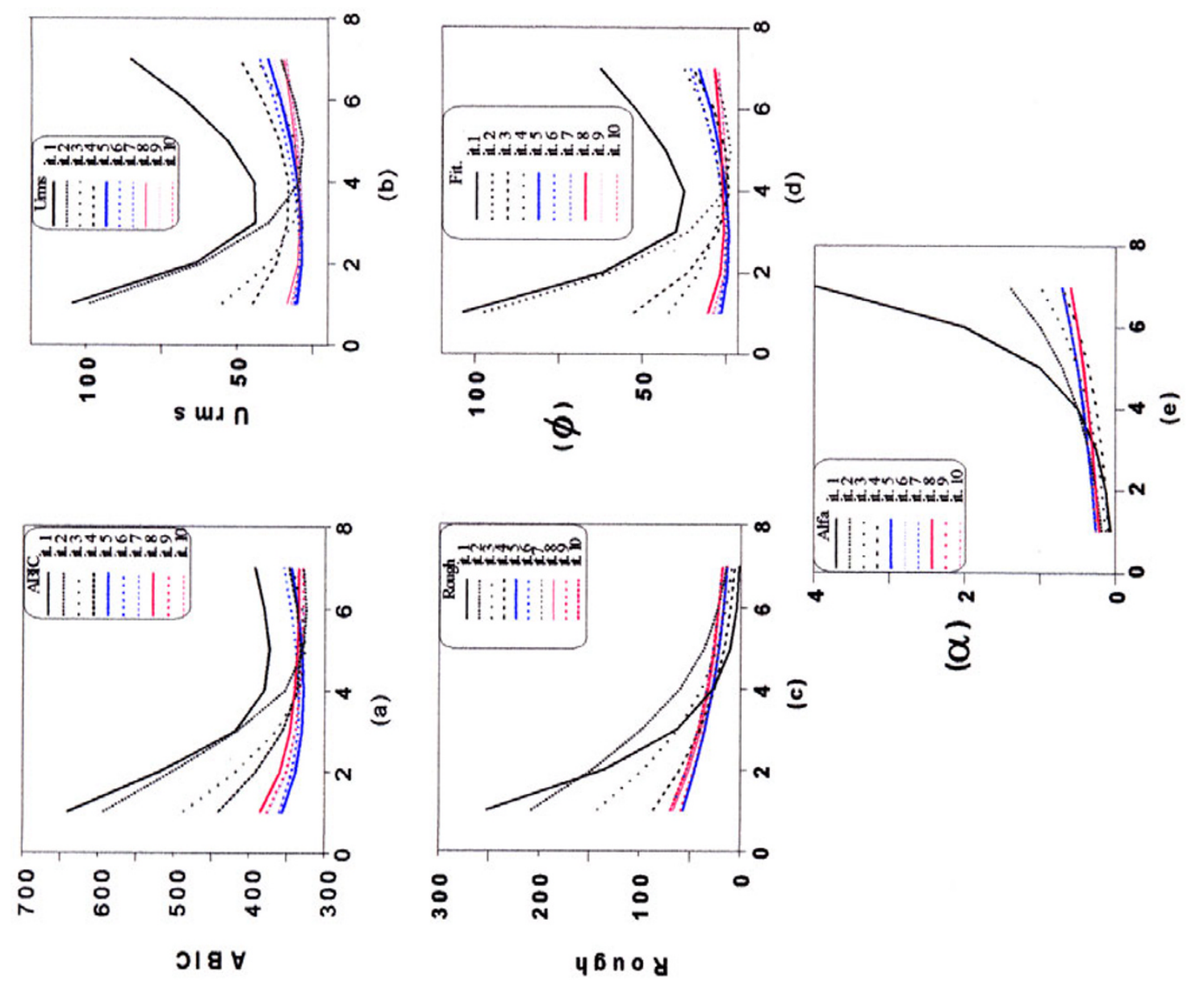


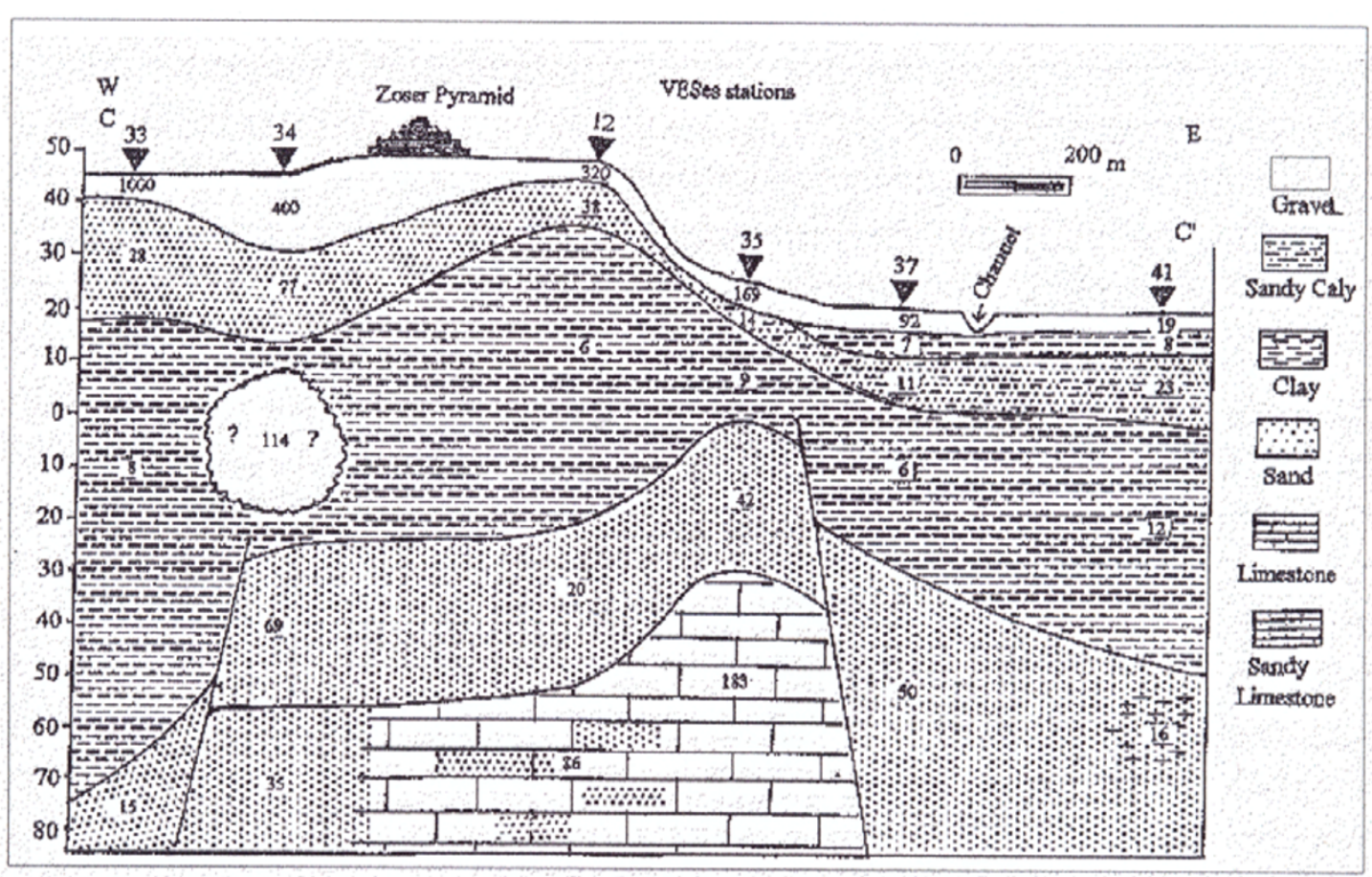

(a): 1D Geoelectrical Cross section.

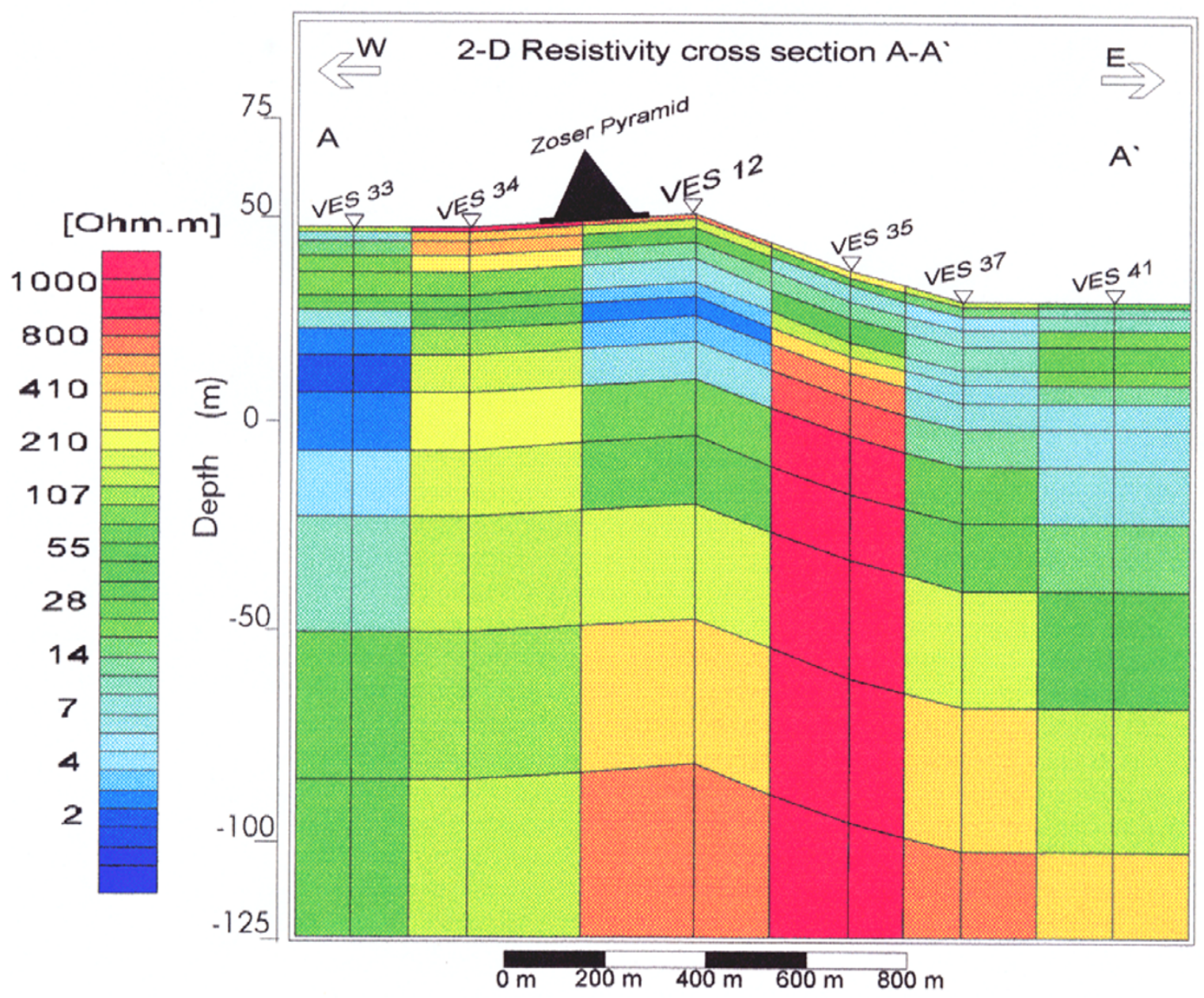

(b): 2D Geoelectrical Cross section.

Fig. 6. The Geoelectrical cross section along the profile A-A'. 


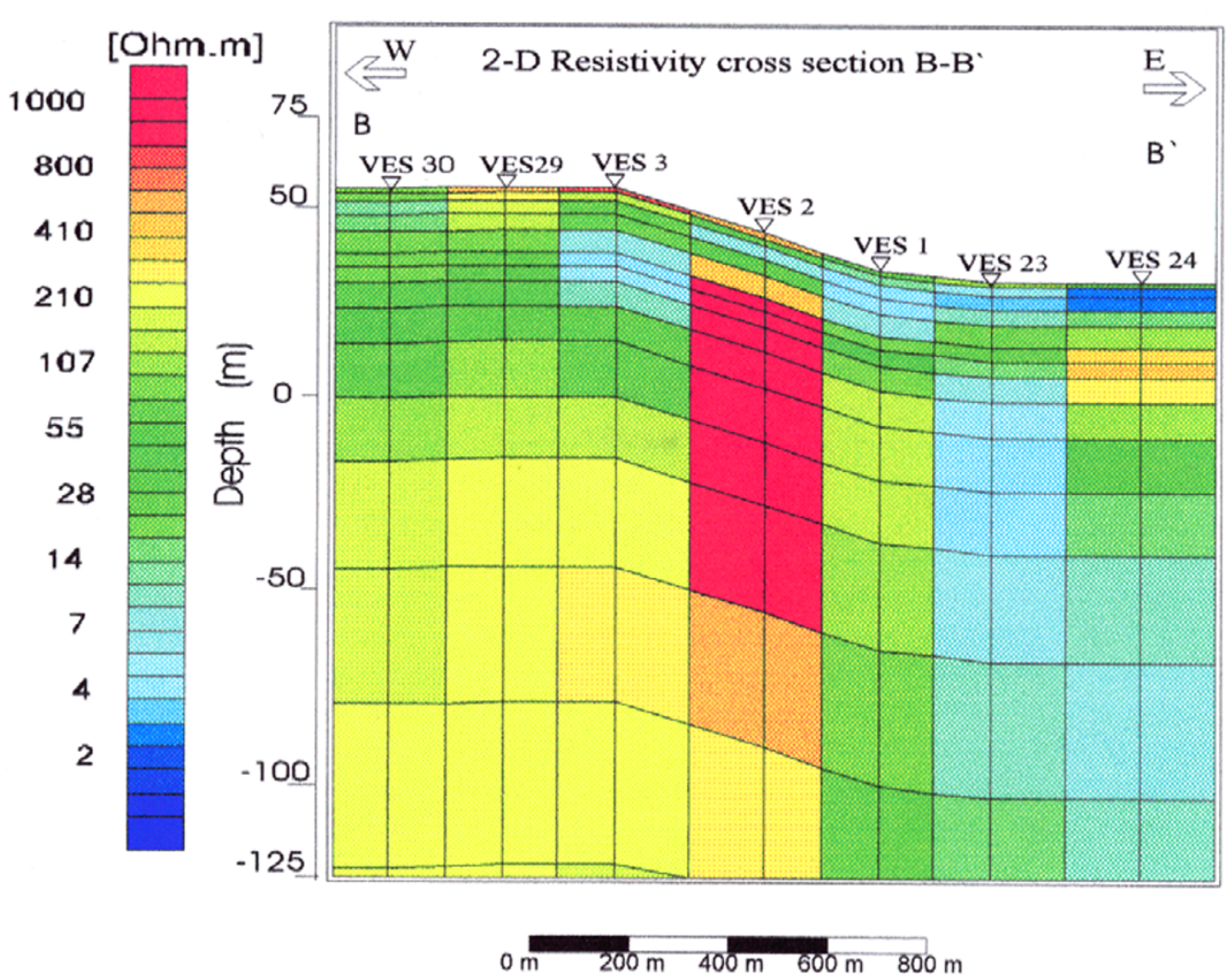

(a)

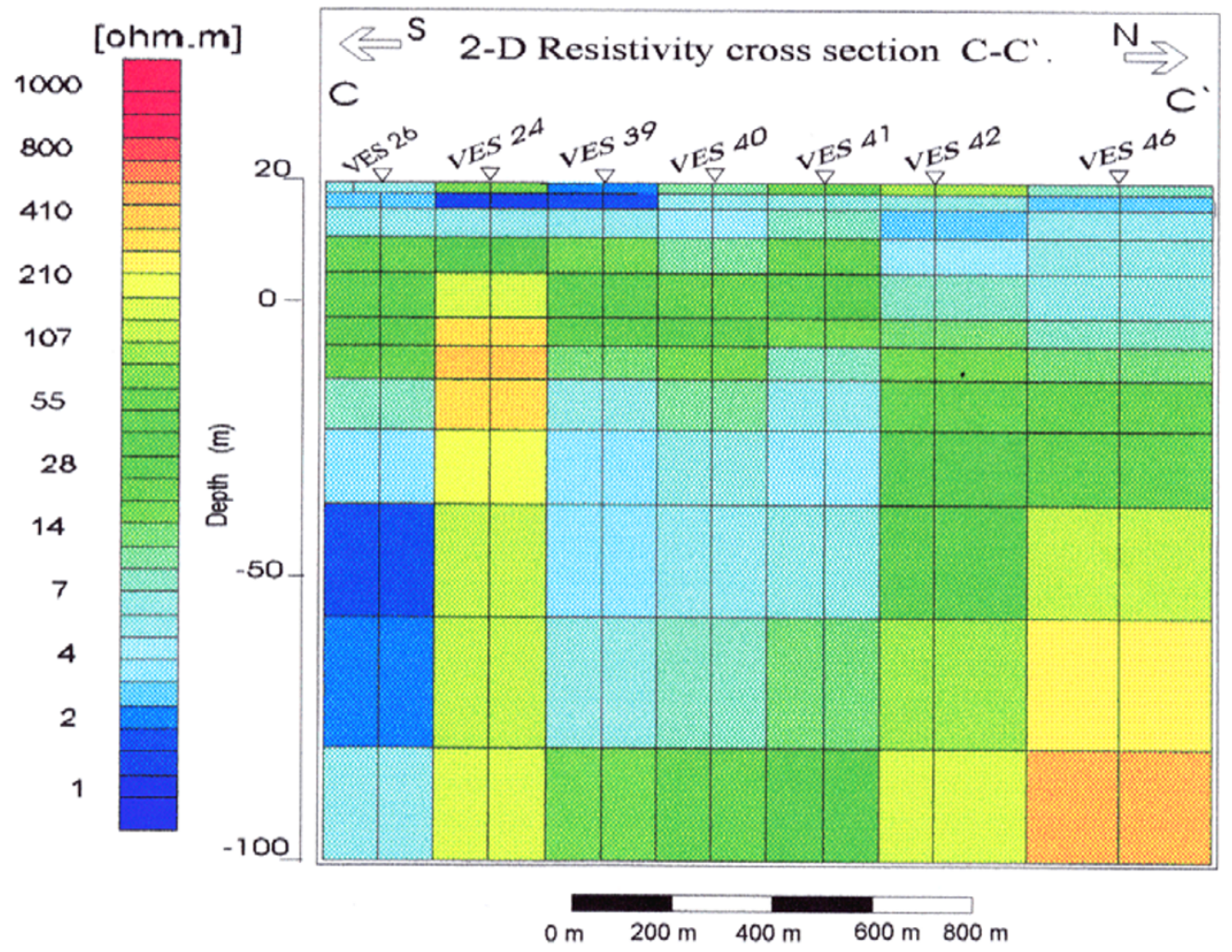

(b)

Fig. 7. The Geoelectrical cross sections along: (a) the profile B-B', (b) the profile C-C'. 
for the inversion was 145 and the number of resistivity blocks is 99 resistivity blocks. The general features of this inverted model; which correlated with 1D cross section are shallow thin resistive layer followed by a conductive relatively thick layer, then a resistive basement. It is obvious that the shallow section of the eastern part is more conductive. This is due to the presence of cultivated land and Nile silt deposits. This part includes a lenticular moderate resistive layer, which represents the shallow water-bearing unit in the area. However, the resistivity increases as far as depth, where Pliocene and Eocene deposits which are varying in lithology between marl, sandstone and limestone. Except at the VES 34 nearby Zoser pyramid, it has a relatively higher resistivity, which disagreed with the neighboring VES stations. However, the 2D respond clearly had been influenced by the expected geological structure correlated with $1 \mathrm{D}$ section. The correlated 2D cross-section still gives an ambiguous interpretation, which may be an archaeological body.

The inverted 2D cross section of the profile (B-B') which results from the 5-th iteration is shown in Fig. 7(a). The smoothing factor is 0.7 , while the number of the observed data is 124 and 117 resistivity block. Inspection of this section showed that, it has nearly the same geoelectrical structure as profile (A-A'), however the eastern shallow lenticular body became more resistive. This is as well as the high resistivity anomaly around VES 2 became smaller in the size.

Figure 7(b), shows the inverted 2D section of the profile C-C'. It is resulted from 166 data point and 117 resistivity block with smoothing factor 0.3 . It is clearly seen that, the cultivated area in the whole profile had affected the $2 \mathrm{D}$ response. This represents a huge thick body of low resistivity. However, there are some spots of moderate resistivity represent the sand lenses of the Nile deposits. We could find a little bit mismatch at the intersection of the profile C-C' with both the profiles A-A' and B-B', although the interpretation gave almost the same geoelectric sequence. This is because the $2 \mathrm{D}$ section is greatly affected by the direction of Schlumberger array spreading whether is it parallel or perpendicular to the section.

Investigations of the constructed 2D cross-sections of all the study area and its correlation with $1 \mathrm{D}$ section are summarized as:

1. Spots of relatively high resistivity values at shallow depth, which may represent shallow gravel, layer.

2. This is followed by a huge thickness conductive body intruded by a relatively moderate resistivity represents the Quaternary Nile sediments. While the moderate resistivity values represent the shallow water-bearing unit in the study area. This, which has the main danger, affected the archaeological sites in the study area.

3. The $2 \mathrm{D}$ response had been influenced by the geological structures in the area such as Saqqara plateau and the archaeological bodies in the area.

\section{Conclusion}

The present work aims to apply 2D inversion on the Schlumberger resistivity data to elucidate the subsurface structure and the ground water distribution in Saqqara area. Forty-seven Schlumberger VES were interpreted in terms of $2 \mathrm{D}$ cross section using algorithm based on ABIC least squares and utilize finite element calculation mesh.

The inversion procedure can reduce the misfit through iterations. However, the resultant cross-section of 2D model often shows a rough spatial resistivity distribution that is, the resistivity changes abruptly between two neighboring blocks, and extraordinary low or high resistivities are obtained. Highly correlation has been found between the resultant 2D cross sections and 1D-inversion results, however it still gives an ambiguous geologic interpretation. It is clear that the $2 \mathrm{D}$ cross section had been affected by spread direction of Schlumberger array as it is appear at the intersection of the profile C-C' with both A-A' and B-B'. So we may recommend the $3 \mathrm{D}$ interpretation for visualizing ground water distribution with depth and to elucidate the subsurface structure of Saqqara area. However, it is recommended to use a different method of 2D analysis, may it gives another solution with a different $2 \mathrm{D}$ response.

A reliable inversion technique for an electrical method is essential to meet the requirement for a more detailed delineation of underground media.

Acknowledgments. The authors would like to express their deepest and sincere thanks to National Research Institute of Astronomy and Geophysics (NRIAG) and Egyptian Supreme council of Antiquities and their staff for a constructive guidance and kind official facilities required for data acquisition in this work. Sincere thanks to all the staff of Exploration Geophysics Lab in Kyushu University for their continuos guidance and support during this work. Moreover, the authors are thankful to the anonymous reviewers and the guest editor Prof. J. Weaver for their constructive criticism, which helped to improve the manuscript to its present form.

\section{References}

Akaike, H., Likelihood and Bayes procedure, in Bayesian Statistics, edited by J. M. Bernardo, et al., pp. 143-166, Univ. press, Valencia, Spain, 1980. Constable, S., L. Robert, and C. Constable, Occam's inversion: A practical algorithm for generating smooth models from EM sounding data, Geophysics, 52(3), 289-300, 1987.

El-Qady, G., Geophysical study for Saqqara area, M.Sc. Thesis, Fac. Sc. Mansoura Univ., Mansoura, Egypt, 1995.

Jackson, D. and M. Matsura, A Baysein approach to a nonlinear inversion, J. Geophys. Res., 90(B1), 581-591, 1985.

Said, R., Subsurface geology of Cairo area: Memories de L., Inst. D’Egypte, Tome soxante, Le Caire, 70 pp., 1975.

Said, R., The Geology of Egypt, 734 pp., Balkema Pub., Roterdam, Netherlands, 1990.

Sasaki, Y., Automatic interpretation of resistivity sounding data over two dimensional structures (I), Geophys. Explor. of Japan (Butsuri Tanko), 34, 341-350, 1981 (in Japanese).

Strenberg, B. K., Electrical resistivity of the crust in the southern extension of the Canadian Shield-layered earth models, J. Geophys. Res., 84, 212-228, 1979.

Uchida, T., Two-Dimensional resistivity inversion for Schlumberger sounding, Geophys. Explor. of Japan (Butsuri Tansa), 44(1), 1-17, 1991.

Uchida, T., Smooth 2-D inversion for Magnetotelluric Data Based on Statistical Criterion ABIC, J. Geomag. Geoelectr., 45, 841-858, 1993.

Vozoff, K. and D. Jupp, Joint invesrion of geophysical data, Geophys. J. R. Astr. Soc., 42, 977-991, 1975.

Zohdy, A. R., A new method for the automatic interpretation of Schlumberger and Wenner sounding curves, Geophysics, 54(2), 245-253, 1989.

G. El-Qady (e-mail: Gad@mine.kyushu-u.ac.jp), C. Sakamoto, and K. Ushijima 